Check for updates

Cite this: RSC Adv., 2017, 7, 44680

Received 14th August 2017

Accepted 13th September 2017

DOI: $10.1039 / c 7 r a 08990 b$

rsc.li/rsc-advances

\title{
A short synthesis of 7-amino alkoxy homoisoflavonoides $\dagger$
}

\author{
Ali Asadipour, ${ }^{a}$ Yaghoub Pourshojaei, (DD *ab Khalil Eskandari (D) *a \\ and Alireza Foroumadic
}

The synthesis of novel derivatives of homoisoflavonoids as potentially interesting medicinally important heterocycles in an efficient catalytic two step route is introduced. In the first step, 7aminoalkoxychromane-4-ones are synthesized via reaction between 7-hydroxychroman-4-one and aminoethylchlorides in the presence of potassium carbonate as a Brønsted base catalyst. In the next step, obtained 7-aminoalkoxychromane-4-ones are reacted with a wide range of arylaldehydes in the presence of hydrochloric acid as Brønsted acid catalyst to obtain homoisoflavonoids. Target products are medicinally very important heterocycles, because their analogs show cytotoxic activities towards human cancer cell lines.

\section{Introduction}

Homoisoflavonoids are a class of flavonoid-type natural products which have been isolated from numerous plants, ${ }^{1}$ notably in the Hyacinthaceae family. ${ }^{2}$ Their chemical structures consist of two aromatic carbocycles (phenyl) and one non-aromatic heterocycle (dihydropyran-4-one). The homoisoflavonoids can be divided into five categories: the 3-benzyl-4-chromanones (A), the 3-hydroxy-3-benzyl-4-chromanones (B), the 3-benzyl-chrom2-en-4-ones (C), the scillascillins (D), and the 3-benzylidene-4chromanones (E or Z) (E) (Fig. 1). ${ }^{3}$

To date, a wide range of biological behaviors of homoisoflavonoids, their synthesis and biosynthesis have been studied and reported in the literature. ${ }^{\mathbf{1 , 4}}$ These types of compounds have shown many biological activities such as antiangiogenic, ${ }^{5}$ antifungal, ${ }^{6}$ hypocholesterolemic, ${ }^{7}$ antimutagenic, ${ }^{8}$ anti-inflammatory, ${ }^{9}$ and antiviral activities. ${ }^{\mathbf{1 0 , 1 1}}$

Among natural products containing homoisoflavonoid scaffold, Bonducellin (isolated from Caesalpinia bonducella and Caesalpinia pulcherrima) ${ }^{\mathbf{1 2}}$ acts as inhibitor of multidrug resistance efflux pump. ${ }^{13}$ Also, isobonducellin, (the $Z$-isomer of Bonducellin), and 8-methoxybonducellin (isolated from C. pulcherrima $)^{\mathbf{1 4 , 1 5}}$ have been shown anti-oxidant activity. ${ }^{\mathbf{1 6}}$ In addition, Sappanone A (isolated from the heartwood of Caesalpinia)

${ }^{a}$ Department of Medicinal Chemistry, Faculty of Pharmacy \& Pharmaceutics Research Center, Institute of Neuropharmacology, Kerman University of Medical Sciences, Kerman, Iran.E-mail: y.pourshoja@kmu.ac.ir; khalileskandari@yahoo.com

${ }^{b}$ Neuroscience Research Center, Institute of Neuropharmacology, Kerman University of Medical Sciences, Kerman, Iran

${ }^{c}$ Drug Design and Development Research Center, Tehran University of Medical Sciences, Tehran, Iran

$\dagger$ Electronic supplementary information (ESI) available. See DOI: 10.1039/c7ra08990b is another homoisoflavonoid type compounds which exhibits antioxidant activity (Fig. 2). ${ }^{17}$

Considering above and existence the great interest in synthesis of important pharmacological active heterocycles, ${ }^{\mathbf{1 8}}$ the synthesis of homoisoflavonoids has also attracted the interest of chemists and pharmaceutics.

Nowadays, there are numerous successful attempts to the laboratory synthesis of these kinds of compounds. In general, there are two protocols for the synthesis of these compounds. In the first approach, homoisoflavonoids are obtained from condensation reaction between 4-chromanones and arylaldehydes in methanol as solvent and by passing $\mathrm{HCl}$ gas as Brønsted acid catalyst or by using piperidine as a Brønsted base<smiles>O=C1c2ccccc2OC[C@H]1Cc1ccccc1</smiles>

A<smiles>O=c1c(Cc2ccccc2)coc2ccccc12</smiles><smiles>O=C1c2ccccc2OC[C@]1(O)Cc1ccccc1</smiles>

B<smiles>O=C1c2ccccc2OCC12Cc1ccccc1C2</smiles>

D<smiles>O=C1C(=Cc2ccccc2)COc2ccccc21</smiles>

Fig. 1 Chemical structure classification of homoisoflavonoids. 
<smiles>COc1ccc(/C=C2\COc3cc(O)ccc3C2=O)cc1</smiles>

Bonducellin<smiles>COc1ccc(/C=C2\COc3c(ccc(O)c3OC)C2=O)cc1</smiles>

8-methoxybonducellin<smiles>COc1ccc(/C=C2/COc3cc(O)ccc3C2=O)cc1</smiles>

Isobonducellin<smiles>O=C1/C(=C/c2ccc(O)c(O)c2)COc2cc(O)ccc21</smiles>

Sappanone A
Fig. 2 Some selected homoisoflavonoids having pharmacological activity.

$$
\text { (1) }
$$

Scheme 1 Alternative two steps route to the synthesis of 7-hydroxychroman-4-one.

catalyst $^{\mathbf{1 9 , 2 0}}$ followed by isomerisation of the double bond in the presence of $\mathrm{Pd} / \mathrm{C}$ at $250{ }^{\circ} \mathrm{C}$. In second ones homoisoflavonoids are obtained by hydrogenation of chalcones followed by one carbon extension by the use of ethyl formate/sodium ${ }^{21}$ or methanesulfonyl chloride/DMF. ${ }^{22}$ Based on all the above, herein, we wish to report an efficient route to the synthesis of a wide range of novel homoisoflavonoids by rely on first mentioned protocol. By this achievement the scope of homoisoflavonoids as medicinally important molecules was developed.

\section{Results and discussion}

Following to our continuous attempts to develop the new and green strategies for the synthesis of newly prepared organic compounds $^{23}$ especially new chemical intermediates and heterocycles, ${ }^{24,25}$ and also alternative multi-steps routes to the synthesis of medicinally important and biological active heterocycles, ${ }^{26}$ in this study, our attention, is turned towards the synthesis of new homoisoflavonoid derivatives. For this purpose, we first had to prepare 7-hydroxychroman-4-one 4 via a two-step reported method $^{17}$ including the Friedel-Crafts acylation and then intramolecular cyclization (Scheme 1). As can be seen from Scheme 1, at first, $2^{\prime}, 4^{\prime}$-dihydroxy-3-chloro propiophenone $\mathbf{3}$ is synthesized from acylation reaction between resorcinol (1) and 3-chloropropionic acid (2), in the presence of $\mathrm{CF}_{3} \mathrm{SO}_{3} \mathrm{H}$ as catalyst. Then, $2^{\prime}, 4^{\prime}$-dihydroxy-3-chloro propiophenone 3 in the presence of $\mathrm{NaOH}$, undergoes intramolecular cyclization to obtain 7-hydroxychroman-4-one 4.

In the next step, 7-hydroxychroman-4-one $\mathbf{4}$ via a reaction which is well-known as Williamson ether synthesis is reacted with appropriate aminoethylchlorides $\mathbf{5}$ to obtain 7aminoalkoxychroman-4-ones $\mathbf{6}$. The role of $\mathrm{KI}$ in this reaction is activation of aminoethylchloride to nucleophilic attack. Also $\mathrm{K}_{2} \mathrm{CO}_{3}$ has been used as Brønsted catalyst to activation of phenolic $\mathrm{OH}$. After preparation, purification, and characterization of 7-aminoalkoxychroman-4-ones $\mathbf{6}$, they are employed in the reaction with a wide range of arylaldehydes 7 containing both electron donor and electron withdrawing groups via Knoevenagel condensation reaction to give novel homoisoflavonoid derivatives 8 (Scheme 2). The role of $\mathrm{HCl}$ in this reaction is inevitable and acts as Brønsted acid catalyst to activation of both 7-aminoalkoxychroman-4-ones and aryl aldehydes to condense together.

By this achievement, a broad range of novel homoisoflavonoids as important potentially interesting biological active products were synthesized and the scope of these compounds were developed (Table 1).

It was clearly observed that the process can tolerate both electron-withdrawing and electron-donating groups on the phenyl ring. All target products were fully characterized by spectral data (H NMR, C NMR, and IR), along with elemental

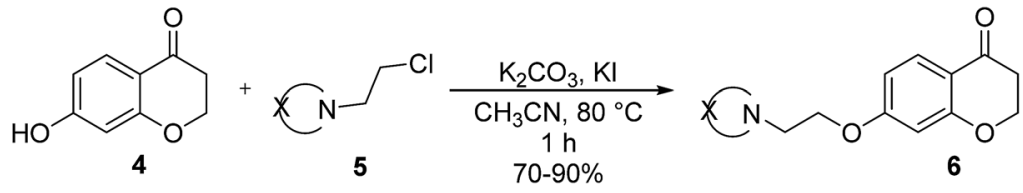

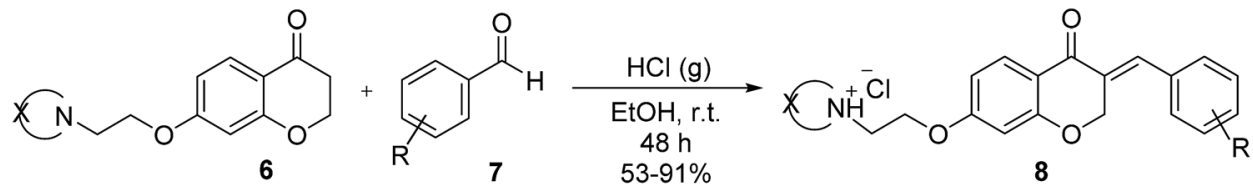

$\mathrm{X}:-\mathrm{CH}_{2} \mathrm{CH}_{2} \mathrm{CH}_{2} \mathrm{CH}_{2} \mathrm{CH}_{2}$ or $-\mathrm{CH}_{2} \mathrm{CH}_{2} \mathrm{OCH}_{2} \mathrm{CH}_{2}-$ R: 4- $\mathrm{NO}_{2}, 3,4,5-\left(\mathrm{OCH}_{3}\right)_{3}, 4-\mathrm{F}, 4-\mathrm{Cl}, 4-\mathrm{OCH}_{3}, 3,4-\left(\mathrm{OCH}_{3}\right)_{2}, 4-\mathrm{CH}_{3}, 3-\mathrm{NO}_{2}, 4-\mathrm{OH}$

Scheme 2 Cascade two step routes to the synthesis of homoisoflavonoid derivatives. 
Table 1 Alternative synthesis of 7-amino alkoxy homoiso-flavonoides

Entry<smiles>O=C1/C(=C/c2ccc([N+](=O)[O-])cc2)COc2cc(OCCN3CCOCC3)ccc21</smiles>

$8 a$<smiles>O=C1/C(=C/c2ccc(F)cc2)COc2cc(OCCN3CCOCC3)ccc21</smiles><smiles>O=C1/C(=C/c2ccc(Cl)cc2)COc2cc(OCCN3CCOCC3)ccc21</smiles><smiles>COc1ccc(/C=C2\COc3cc(OCCN4CCOCC4)ccc3C2=O)cc1</smiles><smiles>Cc1ccc(/C=C2\COc3cc(OCCN4CCOCC4)ccc3C2=O)cc1</smiles><smiles>O=C1/C(=C/c2ccc(O)cc2)COc2cc(OCCN3CCOCC3)ccc21</smiles><smiles>O=C1/C(=C/c2ccc([N+](=O)[O-])cc2)COc2cc(OCCN3CCCCC3)ccc21</smiles>

8<smiles>O=C1/C(=C/c2ccc(F)cc2)COc2cc(OCCN3CCCC3)ccc21</smiles><smiles>O=C1/C(=C/c2ccc(Cl)cc2)COc2cc(OCCN3CCCCC3)ccc21</smiles><smiles>COc1ccc(/C=C2\COc3cc(OCCN4CCCCC4)ccc3C2=O)cc1</smiles>

10
Yield $^{a}(\%)$

M.p. $\left({ }^{\circ} \mathrm{C}\right)$

90

194-196

57

222-223

69

228-230

77

174-176

66

170-172

57

235-237

90

189-192

66

205-207

189-191

174-176 
Table 1 (Contd.)

Entry Product

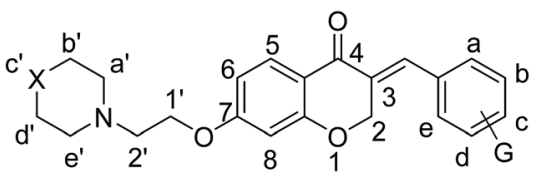

Fig. 3 Atom numbering refers to the order of real atoms in interpretation of NMR data.

analysis. The data of all products were in good agreement with the expected structures (see ESI $\dagger$ ). Also, the numbering of atoms in the target compounds for interpretation of NMR data is indicated in Fig. 3.

In another investigation, we examined other conditions such as using various solvents including $\mathrm{H}_{2} \mathrm{O}, \mathrm{CHCl}_{4}, \mathrm{CH}_{3} \mathrm{CN}$, dioxane, toluene, DMF, DMSO in different temperatures at r.t.,
$40{ }^{\circ} \mathrm{C}, 60{ }^{\circ} \mathrm{C}, 80^{\circ} \mathrm{C}, 100{ }^{\circ} \mathrm{C}$, and $120^{\circ} \mathrm{C}$, and also the effect of other homogeneous and heterogeneous basic and acidic catalysts such as $\mathrm{SSC}, \mathrm{K}_{2} \mathrm{CO}_{3}, \mathrm{NaOH}, \mathrm{KOH}$, piperidine, and triethylamine, $\mathrm{ZnO}, \mathrm{MgCl}_{2}, \mathrm{FeCl}_{3}, \mathrm{CF}_{3} \mathrm{COOH}, \mathrm{CH}_{3} \mathrm{COOH}, \mathrm{H}_{3} \mathrm{PO}_{4}$ on model reaction $(\mathbf{8 j})$ was evaluated. The results obtained from this experiment show that only using $\mathrm{HCl}_{(\mathrm{g})}$ at room temperature in ethanol provides best conditions to obtain our products with highest yields. Because of our target products have containing amine side chain, the use of $\mathrm{HCl}_{(\mathrm{g})}$ in our procedure cause to obtain amine containing $\mathrm{HCl}$ as salt products which leads to precipitate target products and subsequently results to improve the yield of reaction.

As all of our synthesized products are novel compounds, for comparison of our current approach to others available, we selected compound $\mathbf{8 j}$ as model and compared its obtaining<smiles>COc1ccc(/C=C2\COc3cc(OCCN4CCCC4)ccc3C2=O)cc1</smiles>

$\mathrm{HCl}, \mathrm{EtOH}$, r.t., 83\% [this work, 8j]<smiles>COc1ccc(/C=C2\COc3ccccc3C2=O)cc1</smiles>

$\mathrm{HCl}, \mathrm{EtOH}$, r.t., $61 \%[27]$<smiles>COc1ccc2c(c1)OC/C(=C\c1ccc([OH+])cc1)C2=O</smiles>

$\mathrm{KOH}(4 \%) / \mathrm{EtOH}$, r.t., $42 \%[30]$<smiles>COc1ccc(/C=C2\COc3c(ccc(O)c3O)C2=O)cc1</smiles>

Piperidine, $80^{\circ} \mathrm{C}, 60 \%$ [32]<smiles>COc1ccc(/C=C2\COc3ccccc3C2=O)c(O)c1</smiles>

$\mathrm{HCl}$, EtOH, r.t., 56\% [28]<smiles>COc1ccc(C=C2COc3ccccc3C2=O)cc1</smiles>

$\mathrm{NaOH}, \mathrm{EtOH}$, r.t., $73 \%$ [31]<smiles>COc1ccc(/C=C2\COc3cc(O)ccc3C2=O)cc1</smiles>

Piperidine, $80^{\circ} \mathrm{C}, 44 \%$ [33]<smiles>COc1ccc(/C=C2\COc3ccccc3C2=O)cc1</smiles>

$\mathrm{H}_{3} \mathrm{PO} 4,80^{\circ} \mathrm{C}, 69 \%[29]$<smiles>COc1ccc(/C=C2\CCc3ccccc3C2=O)cc1</smiles>

$\mathrm{NaOH}, \mathrm{EtOH}$, r.t., $78 \%$ [27]<smiles>COc1cc(OC)c(/C=C2\COc3cc(O)ccc3C2=O)c(OC)c1</smiles>

Piperidine, $110^{\circ} \mathrm{C}, 42 \%[34]$

Scheme 3 Comparison of homoisoflavonoids synthesis under different conditions with our current protocol. 
method with other homoisoflavonoids containing 4-OR substituted on benzylidene moiety under different conditions (Scheme 3). The results show that best and more efficient condition to the synthesis of homoisoflavonoids is provided by using $\mathrm{HCl}$ in ethanol at room temperature which leads to achieve the product with highest yield than others. ${ }^{27-34}$

Based on the above, by this achievement, the scope of homoisoflavonoids as very medicinally important compounds was developed. Rapid formation and easily purification of target products, needing a little of $\mathrm{HCl}_{(\mathrm{g})}$ to accelerate reaction can be countered as other advantages of this protocol. However, this protocol tolerate some disadvantages such as using $\mathrm{HCl}_{(\mathrm{g})}$ as hazardous material, the excessing use of $\mathrm{HCl}_{(\mathrm{g})}$ lead to obtain unfavorable products, and working with $\mathrm{HCl}_{(\mathrm{g})}$ needs to observe more safety principles in the laboratory.

\section{Experimental}

All reagents, starting materials, and solvents applied in this work were purchased from commercial Merck AG, Aldrich, and Acros Organics chemical companies. Melting points of compounds were determined on Kolfer hot stag apparatus and are uncorrected. IR spectra were taken using Nicolet FT-IR Magna 550 spectrographs on $\mathrm{KBr}$ plates. The NMR spectra were recorded on Bruker Avance 400 or $500 \mathrm{MHz}$, FT-NMR spectrometers, Germany. ${ }^{1} \mathrm{H}$ NMR data are reported in the following order: chemical shifts $(\delta)$ in part per million (ppm) down field from TMS as internal standard; approximate coupling constant values $(J)$ in Hertz (HZ); spin multiplicities (s, singlet; d, doublet; t, triplet; m, multiplet; q, quartet; br, broad; quin, quintet). Thin-layer chromatography (TLC) was performed on pre-coated Silica Gel F254 plates for controlling the reaction progress.

\section{Typical procedure to the synthesis of $2^{\prime}, 4^{\prime}$-dihydroxy-3-chloro} propiophenone $3^{35}$

Trifluoromethanesulfonic acid (100 g, $0.67 \mathrm{~mol})$ was added to a stirred mixture of resorcinol (1) $(20.0 \mathrm{~g}, 0.182 \mathrm{~mol})$ and 3chloropropionic acid (2) (20.0 g, $0.184 \mathrm{~mol})$ at room temperature. It was allow to solution reaching $80{ }^{\circ} \mathrm{C}$ for $30 \mathrm{~min}$, and then cooled to room temperature over $15 \mathrm{~min}$. In the following step, the solution was poured into chloroform $(400 \mathrm{~mL})$, and then slowly poured into water $(400 \mathrm{~mL})$. Afterwards, the layers were separated from each other. The aqueous phase was extracted with $2 \times 200 \mathrm{~mL}$ of $\mathrm{CHCl}_{3}$. The combined organic phases were washed with brine, dried over $\mathrm{Na}_{2} \mathrm{SO}_{4}$, and finally filtered. The highly viscous product was obtained by concentration in vacuum as an orange semisolid $(24.1 \mathrm{~g}, 66 \%) .{ }^{1} \mathrm{H}-$ NMR $\delta 12.56(\mathrm{lH}, \mathrm{s}), 7.63(\mathrm{lH}, \mathrm{d}, J=7.6 \mathrm{~Hz}), 6.37-6.46(2 \mathrm{H}$, $\mathrm{m}), 3.92(2 \mathrm{H}, \mathrm{t}, J=6.3 \mathrm{~Hz}), 3.41(2 \mathrm{H}, \mathrm{t}, J=6.3 \mathrm{~Hz})$.

\section{Typical procedure to the synthesis of 7-hydroxychroman-4-one $4^{35}$}

$2^{\prime}, 4^{\prime}$-Dihydroxy-3-chloro propiophenone 3 (24.1 g, $0.12 \mathrm{~mol}$ ) was added to a stirred solution of $\mathrm{NaOH}(2 \mathrm{M}, 1.0 \mathrm{~L})$ at $5{ }^{\circ} \mathrm{C}$. Then the solution was allowed to warm around room temperature over
$2 \mathrm{~h}$. Afterwards, the reaction mixture was re-cooled to $5{ }^{\circ} \mathrm{C}$, and by the use of $\mathrm{H}_{2} \mathrm{SO}_{4}(6 \mathrm{M}, 100 \mathrm{~mL})$, the $\mathrm{pH}$ was adjusted to 2 . At the end, the mixture was extracted three times with EtOAc $(3 \times$ $200 \mathrm{~mL}$ ), washed with brine, dried over $\mathrm{Na}_{2} \mathrm{SO}_{4}$, and then filtered. The crude product was obtained by concentration in vacuum as a tan solid. The crude product was softened by hexanes, filtrated, and then recrystallized by water to give colorless crystals $(18.4 \mathrm{~g}, 61 \%)$ : mp: $143{ }^{\circ} \mathrm{C} ;{ }^{1} \mathrm{H}-\mathrm{NMR} \delta 7.84(\mathrm{lH}$, $\mathrm{d}, J=9.4 \mathrm{~Hz}), 6.73(1 \mathrm{H}$, brd s), $6.55(1 \mathrm{H}, \mathrm{dd}, J=9.4,1.8 \mathrm{~Hz}), 6.43$ $(1 \mathrm{H}, \mathrm{d}, J=1.8 \mathrm{~Hz}), 4.54(2 \mathrm{H}, \mathrm{t}, J=6.5 \mathrm{~Hz}), 2.78(2 \mathrm{H}, \mathrm{t}, J=6.5$ $\mathrm{Hz})$; IR ( $\left.\mathrm{cm}^{-1}\right)$ : 3160, 1660, 1596, 1451, 1370, 1254.

\section{General procedure for the synthesis of 7-(2-aminoethoxy) chroman-4-ones $6^{23 b}$}

To a mixture of 7-hydroxychroman-4-one 1 (1 $\mathrm{mmol})$ and appropriate aminoethyl chloride hydrochloride $(1.1 \mathrm{mmol})$ in acetonitrile $(10 \mathrm{~mL})$ in a single-neck round bottom flask, potassium carbonate $(3 \mathrm{mmol})$ and a catalytic amount of potassium iodide $(5 \% \mathrm{~mol})$ were added. The mixture was stirred under reflux for 10-14 h and regularly monitored for reaction progress by TLC using $10 \%$ methanol/dichloromethane as the solvent system. After cooling down to room temperature, the entire content was filtered under suction and the solid inorganic salts were washed with acetonitrile $(3 \times 10 \mathrm{~mL})$. The resulting filtrate was evaporated to dryness under reduced pressure and residue was purified by silica column chromatography (1-9\% methanol in dichloromethane, $v / v$ as eluent) to afford compounds 6 in $70-90 \%$ yields.

General procedure for the synthesis of $E$-7-(2-aminoethoxy)-3aryliden-2,3-dihydrochromen-4-one hydrochloride derivatives 8

A solution of 7-aminoalkoxychroman-4-ones 6 (1 mmol), and appropriate arylaldehyde $(1 \mathrm{mmol})$ in $\mathrm{EtOH}(5 \mathrm{~mL})$ was stirred at room temperature for $1 \mathrm{~min}$, under passing a steam of $\mathrm{HCl}$ gas. After the time, the insertation of $\mathrm{HCl}$ was blockade, and the mixture was stirred overnight at room temperature to precipitate product. Subsequently, filter-off the precipitated product and crystallized from EtOH to give compounds 8. In some cases which the crystals need to further purification, silica gel column chromatography with ethyl acetate/n-hexane $(1: 2)$ as eluent was applied to obtain crystalline pure products.

\section{Typical procedure for the synthesis of $E$-7-(2-morpholino- ethoxy)-3-(4-nitrobenzyliden)-2,3-dihydrochromen-4-one hydrochloride $8 \mathrm{a}$}

To a stirred mixture of 7-morpholinoethoxychroman-4-one 6 $(0.277 \mathrm{~g}, 1 \mathrm{mmol})$ in $\mathrm{EtOH}(5 \mathrm{~mL})$ at room temperature equipped with bypassing $\mathrm{HCl}_{(\mathrm{g})}$ valve, 4-nitrobenzaldehyde 7a $(0.151 \mathrm{~g}, 1$ mmol) was added. Afterwards, it was permitted to $\mathrm{HCl}_{(\mathrm{g})}$ to insert in reaction mixture for $1 \mathrm{~min}$. When inserting of $\mathrm{HCl}$ to the mixture was completed, it was allowed to the mixture to remain overnight at room temperature to precipitate product. Then, the mixture was filtered, and obtained crude product was recrystallized in boiling EtOH to afford crystalline pure 8a. The obtained product was characterized by IR, ${ }^{1} \mathrm{H}$ NMR, ${ }^{13} \mathrm{C}$ NMR and elemental analysis (C, $\mathrm{H}$ and $\mathrm{N}$ ). 


\section{Spectral data of product 8}

(E)-3-(4-Nitrobenzyliden)-7-(2-morpholinoethoxy)-2,3-dihydro chromen-4-one hydrochloride (8a). FT-IR $\left(\mathrm{KBr}, \mathrm{cm}^{-1}\right) \nu_{\text {max }}: 3374$ $(\mathrm{N}-\mathrm{H}), 1654(\mathrm{C}=\mathrm{O}), 1613\left(\mathrm{C}=\mathrm{C}\right.$ alkene); ${ }^{1} \mathrm{H}$ NMR $\left(\mathrm{CDCl}_{3}, 500\right.$ MHz) $\delta: 8.31$ (d, 2H, H-a, $J=8 \mathrm{~Hz}), 7.97$ (d, 1H, H-6, $J=8 \mathrm{~Hz}$ ), 7.83 (s, 1H, H-8), 7.46 (d, 2H, H-b, $J=8 \mathrm{~Hz}$ ), 6.68 (d, 1H, H-5, J=8 $\mathrm{Hz}$ ), 6.24 (s, 1H, vinylic-H), 5.28 (s, 2H, H-2), 4.16 (br s, $2 \mathrm{H}, \mathrm{H}_{-1}^{\prime}$ ), 3.74 (br, $4 \mathrm{H}, \mathrm{H}-\mathrm{b}^{\prime}$ ), 2.82 (br s, $2 \mathrm{H}, \mathrm{H}-2^{\prime}$ ), 2.58 (br s, $4 \mathrm{H}, \mathrm{H}-\mathrm{a}^{\prime}$ ); ${ }^{13} \mathrm{C}$ NMR (125 MHz, $\mathrm{CDCl}_{3}$ ) $\delta: 180.1,165.5,163.1,147.7,140.9,133.9$, 133.7, 130.4, 129.8, 123.9, 115.7, 115.5, 111.2, 101.5, 67.4, 66.8, 66.4, 57.2, 54.0. Anal. calcd for $\mathrm{C}_{22} \mathrm{H}_{22} \mathrm{~N}_{2} \mathrm{O}_{6} \cdot \mathrm{HCl}$ (446.88): C, 59.13; H, 5.19; N, 6.27. Found: C, 59.18; H, 5.21; N, 6.29.

(E)-3-(4-Fluorobenzyliden)-7-(2-morpholinoethoxy)-2,3-dihydro chromen-4-one hydrochloride (8b). IR $\left(\mathrm{KBr}, \mathrm{cm}^{-1}\right) \nu_{\text {max }}: 3348(\mathrm{~N}-$ $\mathrm{H}), 1662(\mathrm{C}=\mathrm{O}), 1614$ ( $\mathrm{C}=\mathrm{C}$ alkene); ${ }^{1} \mathrm{H}$ NMR (DMSO-d ${ }_{6}, 400$ $\mathrm{MHz}), \delta: 11.56(\mathrm{~s}, 1 \mathrm{H}, \mathrm{NH}), 7.87$ (d, 1H, H-5, J=8.8 Hz), 7.72 (s, $1 \mathrm{H}, \mathrm{H}-8), 7.53$ (dd, $1 \mathrm{H}, \mathrm{H}-\mathrm{Ar}, J=5.6$ and $3.2 \mathrm{~Hz}), 7.34(\mathrm{~m}, 2 \mathrm{H}, \mathrm{H}-$ $\mathrm{Ar}), 6.79(\mathrm{dd}, 1 \mathrm{H}, \mathrm{H}-6, J=8.8$ and $2.4 \mathrm{~Hz}), 6.67$ (d, $1 \mathrm{H}$, vinylic- $\mathrm{H}, J$ $=2.2 \mathrm{~Hz}), 5.41(\mathrm{~d}, 2 \mathrm{H}, \mathrm{H}-2, J=2.2 \mathrm{~Hz}), 4.55\left(\mathrm{t}, 2 \mathrm{H}, \mathrm{H}-1^{\prime}, J=5 \mathrm{~Hz}\right)$, 3.96-3.82 (br m, 4H, H-b'), 3.56 (br s, 2H, H-2'), 3.47 (br s, 2H, H$\mathrm{a}^{\prime}$ ), 3.20 (br s, $\left.2 \mathrm{H}, \mathrm{H}-\mathrm{a}^{\prime}\right) .{ }^{13} \mathrm{C}$ NMR (100 MHz, DMSO-d $\left.{ }_{6}\right) \delta: 180.2$, 164.3, 163.0, 161.8, 135.4, 133.2, 133.1, 130.9, 129.6, 116.4, 116.2, 116.0, 111.4, 102.4, 68.1, 63.6, 63.2, 55.0, 52.0. Anal. calcd for $\mathrm{C}_{22} \mathrm{H}_{22} \mathrm{FNO}_{4} \cdot \mathrm{HCl}$ (419.87): C, 62.93; H, 5.52; N, 3.34. Found: C, $62.91 ; \mathrm{H}, 5.50 ; \mathrm{N}, 3.34$.

(E)-3-(4-Chlorobenzyliden)-7-(2-morpholinoethoxy)-2,3-dihydro chromen-4-one hydrochloride (8c). IR (KBr, $\left.\mathrm{cm}^{-1}\right) \nu_{\max }: 3435$ $(\mathrm{N}-\mathrm{H}), 1666(\mathrm{C}=\mathrm{O}), 1613\left(\mathrm{C}=\mathrm{C}\right.$ alkene); ${ }^{1} \mathrm{H}$ NMR (DMSO-d 6,400 MHz), $\delta: 11.47$ (br s, 1H, NH), 7.87 (d, 1H, H-5, J=8.8 Hz), 7.71 (s, $1 \mathrm{H}, \mathrm{H}-8), 7.55$ (d, $2 \mathrm{H}, \mathrm{H}-\mathrm{a}, J=8.4 \mathrm{~Hz}), 7.50$ (d, 2H, H-b, $J=8.4 \mathrm{~Hz}$ ), $6.79(\mathrm{dd}, 1 \mathrm{H}, \mathrm{H}-6, J=8.8$ and $2.4 \mathrm{~Hz}$ ), 6.67 (d, $1 \mathrm{H}$, vinylic- $\mathrm{H}, J=2.2$ $\mathrm{Hz}), 5.41$ (d, 2H, H-2, $J=2.2 \mathrm{~Hz}$ ), 4.55 (t, 2H, H-1' $J=4.8 \mathrm{~Hz}$ ), 3.93-3.86 (br d, 4H, H-b'), 3.55 (br s, 2H, H-2'), 3.47 (br s, 2H, H-a'), 3.19 (br s, 2H, H-a'). ${ }^{13} \mathrm{C}$ NMR (100 MHz, DMSO-d 6 ) $\delta: 180.1,164.3$, 163.0, 135.1, 134.7, 133.2, 132.5, 131.7, 129.7, 129.3, 116.0, 111.4, 102.4, 68.1, 63.6, 63.3, 55.0, 52.1. Anal. calcd for $\mathrm{C}_{22} \mathrm{H}_{22} \mathrm{ClNO}_{4} \cdot \mathrm{HCl}$ (436.33): C, 60.56; H, 5.31; N, 3.21. Found: C, 60.57; H, 6.19; N, 3.34.

(E)-3-(4-Methoxybenzyliden)-7-(2-morpholinoethoxy)-2,3dihydro chromen-4-one hydrochloride (8d). FT-IR $\left(\mathrm{KBr}, \mathrm{cm}^{-1}\right)$ $\nu_{\max }: 3417(\mathrm{~N}-\mathrm{H}), 1728(\mathrm{C}=\mathrm{O}), 1663\left(\mathrm{C}=\mathrm{C}\right.$ alkene); ${ }^{1} \mathrm{H}$ NMR $\left(\mathrm{DMSO}_{6}, 400 \mathrm{MHz}\right) \delta: 11.51(\mathrm{~s}, 1 \mathrm{H}, \mathrm{NH}), 7.85$ (d, 1H, H-5, J= $8.8 \mathrm{~Hz}$ ), 7.68 (s, 1H, H-8), 7.44 (d, 2H, H-a, $J=8.4 \mathrm{~Hz}$ ), 7.07 (d, $2 \mathrm{H}, \mathrm{H}-\mathrm{b}, J=8.4 \mathrm{~Hz}$ ), 6.77 (dd, $1 \mathrm{H}, \mathrm{H}-6, J=8.8$ and $2 \mathrm{~Hz}$ ), 6.65 (d, $1 \mathrm{H}$, vinylic- $\mathrm{H}, J=2 \mathrm{~Hz}), 5.43(\mathrm{~d}, 2 \mathrm{H}, \mathrm{H}-2, J=2 \mathrm{~Hz}), 4.54(\mathrm{br} \mathrm{s}, 2 \mathrm{H}$, H-1'), 3.92 (br s, 4H, H-b'), 3.82 (s, 3H, -OMe), 3.55 (br s, 2H, H$2^{\prime}$ ), 3.47 (br s, 2H, H-a'), 3.19 (br s, $\left.2 \mathrm{H}, \mathrm{H}-\mathrm{a}^{\prime}\right) .{ }^{13} \mathrm{C} \mathrm{NMR}(100 \mathrm{MHz}$, DMSO-d $\left.{ }_{6}\right) \delta: 180.2,164.1,162.8,136.4,132.2,132.1,129.6$, 129.1, 128.9, 126.8, 116.2, 114.8, 111.2, 102.3, 68.3, 67.9, 63.6, $63.2,55.9,55.0,52.1$. Anal. calcd for $\mathrm{C}_{23} \mathrm{H}_{25} \mathrm{ClNO}_{5} \cdot \mathrm{HCl}$ (431.91): C, 63.96; H, 6.07; N, 3.24. Found: C, 63.97; H, 6.12; N, 3.31.

(E)-3-(4-Methylbenzyliden)-7-(2-morpholinoethoxy)-2,3-dihydro chromen-4-one hydrochloride (8e). IR $\left(\mathrm{KBr}, \mathrm{cm}^{-1}\right) \nu_{\text {max }}: 3390(\mathrm{~N}-$ $\mathrm{H}), 1659(\mathrm{C}=\mathrm{O}), 1603$ (C=C alkene); ${ }^{1} \mathrm{H}$ NMR (DMSO-d ${ }_{6}, 400$ $\mathrm{MHz}), 11.38$ (s, 1H, NH), 7.86 (d, 1H, H-5, J= $8.8 \mathrm{~Hz}$ ), 7.69 (s, 1H, H-8), 7.35 (d, 2H, H-a, J= 8.4 Hz), 7.32 (d, 2H, H-b, J= 8.4 Hz), 6.77 (dd, $1 \mathrm{H}, \mathrm{H}-6, J=8.8$ and $2.4 \mathrm{~Hz}), 6.66(\mathrm{~d}, 1 \mathrm{H}$, vinylic- $\mathrm{H}, J=2 \mathrm{~Hz}$ ), 5.42 (d, 2H, H-2, $J=2 \mathrm{~Hz}), 4.53\left(\mathrm{t}, 2 \mathrm{H}, \mathrm{H}-1^{\prime}, J=5 \mathrm{~Hz}\right.$ ), 3.92 (br s, $\left.4 \mathrm{H}, \mathrm{H}-\mathrm{b}^{\prime}\right), 3.55$ (t, 2H, H-2' $J=5 \mathrm{~Hz}$ ), 3.45 (br s, 2H, H-a'), 3.20 (br s, 2H, H-a'), 2.37 (s, 3H, $\left.-\mathrm{CH}_{3}\right) .{ }^{13} \mathrm{C}$ NMR (100 MHz, DMSO-d 6 ) $\delta: 180.3,164.2,162.9,140.1,136.5,131.5,130.8,130.3,129.9$, 129.6, 116.1, 111.3, 102.4, 68.2, 63.6, 63.2, 55.0, 52.1, 21.5. Anal. calcd for $\mathrm{C}_{23} \mathrm{H}_{25} \mathrm{NO}_{4} \cdot \mathrm{HCl}$ (415.91): C, 66.42; H, 6.30; N, 3.37. Found: C, 66.66; H, 6.39; N, 3.39.

(E)-3-(4-Hydroxybenzyliden)-7-(2-morpholinoethoxy)-2,3dihydro chromen-4-one hydrochloride $(8 \mathrm{f}) . \mathrm{IR}\left(\mathrm{KBr}, \mathrm{cm}^{-1}\right) \nu_{\max }$ : $3077(\mathrm{~N}-\mathrm{H}), 1670(\mathrm{C}=\mathrm{O}), 1582\left(\mathrm{C}=\mathrm{C}\right.$ alkene); ${ }^{1} \mathrm{H}$ NMR (DMSO$\left.\mathrm{d}_{6}, 500 \mathrm{MHz}\right), 11.54$ (s, 1H, NH), 10.22 (s, 1H, OH), 7.83 (d, 1H, $\mathrm{H}-5, J=8.8 \mathrm{~Hz}$ ), 7.63 (s, 1H, H-8), 7.32 (d, 2H, H-a, $J=8.6 \mathrm{~Hz}$ ), $6.90(\mathrm{~d}, 2 \mathrm{H}, \mathrm{H}-\mathrm{b}, J=8.6 \mathrm{~Hz}), 6.76(\mathrm{dd}, 1 \mathrm{H}, \mathrm{H}-6, J=8.8$ and 2.35 $\mathrm{Hz}), 6.64$ (d, 1H, vinylic- $\mathrm{H}, J=2 \mathrm{~Hz}), 5.41$ (d, $2 \mathrm{H}, \mathrm{H}-2, J=2 \mathrm{~Hz}$ ), 4.54 (br s, $2 \mathrm{H}, \mathrm{H}-1^{\prime}$ ), 3.96-3.81 (br m, 4H, H-b'), 3.56-3.44 (br s, $\left.4 \mathrm{H}, \mathrm{H}-\mathrm{a}^{\prime}\right), 3.22$ (t, 2H, H-2' $\left.J=9.2 \mathrm{~Hz}\right) .{ }^{13} \mathrm{C} \mathrm{NMR}(125 \mathrm{MHz}$, DMSO-d $\left._{6}\right) \delta$ : 179.8, 163.5, 162.3, 159.3, 159.1, 136.4, 132.7, 129.0, 127.4, 124.8, 115.8, 110.6, 101.8, 67.9, 63.1, 62.7, 54.5, 51.6. Anal. calcd for $\mathrm{C}_{22} \mathrm{H}_{23} \mathrm{NO}_{5} \cdot \mathrm{HCl}$ (417.88): C, 63.23; H, 5.79; $\mathrm{N}, 3.35$. Found: C, 63.61; H, 5.88; N, 3.37.

(E)-3-(4-Nitrobenzyliden)-7-((2-piperidine-1-yl)ethoxy)-2,3dihydro chromen-4-one hydrochloride $(8 \mathrm{~g}) . \mathrm{IR}\left(\mathrm{KBr}, \mathrm{cm}^{-1}\right) \nu_{\max }$ : $3355(\mathrm{~N}-\mathrm{H}), 1657$ ( $\mathrm{C}=\mathrm{O}), 1609$ ( $\mathrm{C}=\mathrm{C}$ alkene); ${ }^{1} \mathrm{H}$ NMR (DMSO$\left.\mathrm{d}_{6}, 500 \mathrm{MHz}\right) \delta: 10.55$ (br s, $\left.1 \mathrm{H}, \mathrm{NH}\right), 8.31$ (d, 2H, H-a, $J=6.75$ $\mathrm{Hz}$ ), 7.87 (d, 1H, H-5, J = 8.8 Hz), 7.79 (s, 1H, H-8), 7.72 (d, 2H, $\mathrm{H}-\mathrm{b}, J=6.75 \mathrm{~Hz}$ ), 6.79 (d, $1 \mathrm{H}, \mathrm{H}-6, J=8.8 \mathrm{~Hz}), 6.67$ (s, $1 \mathrm{H}$, vinylic-H), 5.42 (s, 2H, H-2), 4.51 (br s, 2H, H-1'), 3.48 (br s, 4H, H-a ${ }^{\prime}$ ), 2.99 (br s, 2H, H-2'), 1.78-1.37 (br m, 6H, H-c' and $\mathrm{H}-\mathrm{b}^{\prime}$ ). ${ }^{13} \mathrm{C}$ NMR (125 MHz, DMSO-d 6 ) $\delta: 180.1,164.8,163.1,133.9$, 133.7, 130.4 , 130.0, 129.6, 123.9, 123.5, 115.9, 111.0, 101.5, 67.5, 65.7, 55.3, 53.8, 22.6, 22.2. Anal. calcd for $\mathrm{C}_{23} \mathrm{H}_{24} \mathrm{~N}_{2} \mathrm{O}_{5} \mathrm{HCl}$ (444.91): C, 62.09; H, 5.66; N, 6.30. Found: C, 62.26; H, 5.79; N, 6.34 .

(E)-3-(4-Fluorobenzyliden)-7-((2-piperidine-1-yl)ethoxy)-2,3dihydro chromen-4-one hydrochloride $(8 \mathrm{~h}) . \mathrm{IR}\left(\mathrm{KBr}, \mathrm{cm}^{-1}\right) \nu_{\max }$ : $3387(\mathrm{~N}-\mathrm{H}), 1667(\mathrm{C}=\mathrm{O}), 1616\left(\mathrm{C}=\mathrm{C}\right.$ alkene); ${ }^{1} \mathrm{H} \mathrm{NMR}\left(\mathrm{CDCl}_{3}\right.$, $300 \mathrm{MHz}) \delta: 12.53(\mathrm{~s}, 1 \mathrm{H}, \mathrm{NH}), 7.97(\mathrm{br} \mathrm{s}, 1 \mathrm{H}, \mathrm{H}-5), 7.80(\mathrm{~s}, 1 \mathrm{H}, \mathrm{H}-$ 8), 7.30-7.14 (m, 4H, H-a and H-b), 6.63 (br s, 1H, H-6), 6.45 (br s, $1 \mathrm{H}$, vinylic-H), 5.31 (s, 2H, H-2), 4.68 (br s, 2H, H-1'), 3.663.50 (br s, 4H, H-a'), 2.84 (br s, 2H, H-2'), 1.92-1.47 (br m, 6H, H$\mathrm{b}^{\prime}$ and $\left.\mathrm{H}_{-} \mathrm{c}^{\prime}\right) .{ }^{13} \mathrm{C}$ NMR (75 $\mathrm{MHz}, \mathrm{CDCl}_{3}$ ) $\delta: 180.8,164.8,163.3$, $163.0(\mathrm{~d}, J=112.4)$, 136.1, 135.9, 132.0, 131.9, 130.4, 130.1, 116.1, 115.8, 110.7, 102.2, 67.9, 63.6, 55.0, 52.0, 23.0, 22.0. Anal. calcd for $\mathrm{C}_{23} \mathrm{H}_{24} \mathrm{FNO}_{3} \cdot \mathrm{HCl}$ (417.9): C, 66.10; H, 6.03; N, 3.35. Found: C, 66.26; H, 6.14; N, 3.37.

(E)-3-(4-Chlorobenzyliden)-7-((2-piperidine-1-yl)ethoxy)-2,3dihydro chromen-4-one hydrochloride (8i). $\mathrm{IR}\left(\mathrm{KBr}, \mathrm{cm}^{-1}\right) \nu_{\max }$ : $3420(\mathrm{~N}-\mathrm{H}), 1664(\mathrm{C}=\mathrm{O}), 1583\left(\mathrm{C}=\mathrm{C}\right.$ alkene); ${ }^{1} \mathrm{H}$ NMR (DMSO$\left.\mathrm{d}_{6}, 500 \mathrm{MHz}\right) \delta: 10.84(\mathrm{br} \mathrm{s}, 1 \mathrm{H}, \mathrm{NH}), 7.84(\mathrm{~d}, 1 \mathrm{H}, \mathrm{H}-5, J=8.8 \mathrm{~Hz}$ ), 7.69 (s, 1H, H-8), 7.55 (d, 2H, H-a, $J=7.5 \mathrm{~Hz}$ ) 7.48 (d, 2H, H-b, $=7.5 \mathrm{~Hz}), 6.77(\mathrm{~d}, 1 \mathrm{H}, \mathrm{H}-6, J=8.8 \mathrm{~Hz}), 6.65(\mathrm{~s}, 1 \mathrm{H}$, vinylic- $\mathrm{H})$, 5.39 (s, 2H, H-2), 4.51 (br s, 2H, H-1'), 3.47 (br s, 4H, H-a'), 2.98 (br s, 2H, H-2'), 1.78-1.37 (br m, 6H, H-c ${ }^{\prime}$ and $\mathrm{H}^{-} \mathrm{b}^{\prime}$ ). ${ }^{13} \mathrm{C}$ NMR (125 MHz, DMSO-d 6 ) $\delta:$ 179.6, 163.9, 162.6, 134.7, 134.3, 132.8, 132.1, 131.2, 129.2, 128.8, 115.5, 111.0, 101.8, 67.6, 62.9, 
54.3, 52.5, 23.3, 21.2. Anal. calcd for $\mathrm{C}_{23} \mathrm{H}_{24} \mathrm{ClNO}_{3} \cdot \mathrm{HCl}$ (434.36): C, 63.60; H, 5.80; N, 3.22. Found: C, 63.66; H, 6.09; N, 3.34.

(E)-3-(4-Methoxybenzyliden)-7-((2-piperidine-1-yl)ethoxy)2,3-dihydro chromen-4-one hydrochloride $(8 \mathrm{j})$. IR $\left(\mathrm{KBr}, \mathrm{cm}^{-1}\right)$ $\nu_{\text {max }} 3417(\mathrm{~N}-\mathrm{H}), 1728(\mathrm{C}=\mathrm{O}), 1663\left(\mathrm{C}=\mathrm{C}\right.$ alkene); ${ }^{1} \mathrm{H}$ NMR $\left(\right.$ DMSO-d $\left._{6}, 400 \mathrm{MHz}\right) \delta: 11.51(\mathrm{~s}, 1 \mathrm{H}, \mathrm{NH}), 7.85$ (d, 1H, H-5, J= $8.8 \mathrm{~Hz}$ ), 7.68 (s, 1H, H-8), 7.44 (d, 2H, H-a, $J=8.4 \mathrm{~Hz}$ ), 7.07 (d, $2 \mathrm{H}, \mathrm{H}-\mathrm{b}, J=8.4 \mathrm{~Hz}$ ), 6.77 (dd, $1 \mathrm{H}, \mathrm{H}-6, J=8.8$ and $2 \mathrm{~Hz}$ ), 6.65 (d, $1 \mathrm{H}$, vinylic- $\mathrm{H}, J=2 \mathrm{~Hz}), 5.43(\mathrm{~d}, 2 \mathrm{H}, \mathrm{H}-2, J=2 \mathrm{~Hz}), 4.54(\mathrm{br} \mathrm{s}, 2 \mathrm{H}$, H-1'), 3.92 (br s, 4H, H-b'), 3.82 (s, 3H, OMe), 3.55 (br s, 2H, H$2^{\prime}$ ), 3.47 (br s, 2H, H-a'), 3.19 (br s, 2H, H-a'). ${ }^{13} \mathrm{C} \mathrm{NMR}(100 \mathrm{MHz}$, DMSO-d 6 ) $\delta$ : 180.2, 164.1, 162.8, 136.4, 132.2, 132.1, 129.6, 129.1, 128.9, 126.8, 116.2, 114.8, 111.2, 102.3, 68.3, 67.9, 63.6, 63.2, 55.9, 55.0, 52.1. Anal. calcd for $\mathrm{C}_{24} \mathrm{H}_{27} \mathrm{ClNO}_{4} \cdot \mathrm{HCl}$ (431.91): C, 63.96; H, 6.07; N, 3.24. Found: C, 63.97; H, 6.12; N, 3.31.

(E)-3-(4-Methylbenzyliden)-7-((2-piperidine-1-yl)ethoxy)-2,3dihydro chromen-4-one hydrochloride (8k). IR $\left(\mathrm{KBr}, \mathrm{cm}^{-1}\right) \nu_{\max }$ : $3384(\mathrm{~N}-\mathrm{H}), 1664(\mathrm{C}=\mathrm{O}), 1611\left(\mathrm{C}=\mathrm{C}\right.$ alkene); ${ }^{1} \mathrm{H}$ NMR (DMSO$\left.\mathrm{d}_{6}, 500 \mathrm{MHz}\right) \delta: 11.06(\mathrm{br} \mathrm{s}, 1 \mathrm{H}, \mathrm{NH}), 7.84(\mathrm{~d}, 1 \mathrm{H}, \mathrm{H}-5, J=8.6 \mathrm{~Hz})$, 7.68 (s, 1H, H-8), 7.34 (d, 2H, H-a, J= 7.6 Hz), 7.30 (d, 2H, H-b, J $=7.6 \mathrm{~Hz}), 6.76(\mathrm{~d}, 1 \mathrm{H}, \mathrm{H}-6, J=8.6 \mathrm{~Hz}), 6.64(\mathrm{~s}, 1 \mathrm{H}$, vinylic- $\mathrm{H})$, 5.41 (s, 2H, H-2), 4.53 (br s, 2H, H-1'), 3.47 (br s, 2H, H-a'), 3.39 (br s, $2 \mathrm{H}, \mathrm{H}^{-} \mathrm{a}^{\prime}$ ), 2.98 (t, $2 \mathrm{H}, \mathrm{H}-2^{\prime}, J=10.40 \mathrm{~Hz}$ ), 2.35 (s, $3 \mathrm{H}$, $\mathrm{CH}_{3}$ ), 1.84-1.34 (br m, 6H, H-b and $\left.\mathrm{H}-\mathrm{c}^{\prime}\right) .{ }^{13} \mathrm{C}$ NMR $(125 \mathrm{MHz}$, DMSO-d $_{6}$ ) $\delta$ : 180.3, 164.2, 162.9, 140.1, 136.5, 131.5, 130.8, 130.3, 129.9, 129.6, 116.1, 111.3, 102.4, 68.2, 63.6, 63.2, 55.0, 52.1, 21.5. Anal. calcd for $\mathrm{C}_{24} \mathrm{H}_{27} \mathrm{NO}_{3} \cdot \mathrm{HCl}$ (413.94): C, 69.64; $\mathrm{H}$, $6.82 ; \mathrm{N}, 3.38$. Found: C, 66.63; H, 6.69; N, 3.34.

(E)-3-(4-Hydroxybenzyliden)-7-((2-piperidine-1-yl)ethoxy)-2,3dihydro chromen-4-one hydrochloride (81). IR $\left(\mathrm{KBr}, \mathrm{cm}^{-1}\right) \nu_{\max }$ : $3134(\mathrm{~N}-\mathrm{H}), 1666(\mathrm{C}=\mathrm{O}), 1605(\mathrm{C}=\mathrm{C}$ alkene $) ;{ }^{1} \mathrm{H}$ NMR $(300 \mathrm{MHz}$, DMSO-d $\left.{ }_{6}\right) \delta: 10.80(\mathrm{~s}, 1 \mathrm{H}, \mathrm{NH}), 10.24(\mathrm{~s}, 1 \mathrm{H},-\mathrm{OH}), 7.83$ (d, 1H, H-5, $J=8.7 \mathrm{~Hz}$ ), 7.62 (s, 1H, H-8), 7.32 (d, 2H, H-a, $J=7.5 \mathrm{~Hz}), 6.90$ (d, $2 \mathrm{H}, \mathrm{H}-\mathrm{b}, J=7.5 \mathrm{~Hz}$ ), 6.75 (d, 1H, H-6, $J=8.7 \mathrm{~Hz}), 6.62(\mathrm{~s}, 1 \mathrm{H}$, vinylic-H), 5.41 (s, 2H, H-2), 4.50 (br s, 2H, H-1'), 3.44 (br s, $4 \mathrm{H}, \mathrm{H}-$ $\mathrm{a}^{\prime}$ ), 2.97 (br s, 2H, H-2'), 1.78-1.36 (br m, 6H, H-b', H-c ${ }^{\prime}$ ). ${ }^{13} \mathrm{C}$ NMR (75 MHz, DMSO-d ${ }_{6}$ ) $\delta: 180.2,164.0,162.8,159.7,136.9,136.8$, 133.1, 129.6, 127.8, 125.2, 116.2, 116.2, 111.1, 102.2, 68.4, 63.2, 54.8, 53.0, 22.7, 21.6. Anal. calcd for $\mathrm{C}_{23} \mathrm{H}_{25} \mathrm{NO}_{4} \cdot \mathrm{HCl}$ (415.91): C, 66.42; H, 6.30; N, 3.37. Found: C, 66.56; H, 6.34; N, 3.39.

\section{Conclusions}

In summary, for the first time, we presented an efficient route to the synthesis of novel homoisoflavonoids as potentially interesting pharmacological active compounds via a cascade four steps reaction from 7-aminoalkoxychroman-4-ones and arylaldehydes. Medicinally and pharmacologically point of view, with respect to the importance of homoisoflavonoids, by this achievement, the scope of this class of compounds was developed. Also this protocol may be attracted the interest for further investigations and synthetic manipulations to the synthesis and development of medicinally important heterocycles.

\section{Conflicts of interest}

There are no conflicts to declare.

\section{Acknowledgements}

The authors gratefully acknowledge from Pharmaceutics Research Center, Institute of Neuropharmacology, Kerman University of Medical Sciences for partial support of this work.

\section{Notes and references}

1 K. Du-Toit, S. E. Drewes and J. Bodenstein, Nat. Prod. Res., 2010, 24, 457.

2 K. Du-Toit, E. E. Elgorashi, S. F. Malan, D. A. Mulholland, S. E. Drewes and J. Van-Staden, S. Afr. J. Bot., 2007, 73, 236.

3 D. A. Mulholland, S. L. Schwikkard and N. R. Crouch, Nat. Prod. Rep., 2013, 30, 1165.

4 B. M. Abegaz, J. Mutanyatta and M. Nindi, Nat. Prod. Commun., 2007, 2, 475.

5 B. Lee, W. Sun, H. Lee, H. Basavarajappa, R. S. Sulaiman, K. Sishtla, X. Fei, T. W. Corson and S. Y. Seo, Bioorg. Med. Chem. Lett., 2016, 26, 4277.

6 T. Nakib, V. Bezjak, M. J. Meegan and R. Chandy, Eur. J. Med. Chem., 1990, 25, 455.

7 B. S. Kirkiacharian, M. Gomis and P. Koutsourakis, Eur. J. Med. Chem., 1989, 24, 309.

8 M. E. Wall, M. C. Wani, G. Manikumar, H. Taylor and R. McGivney, J. Nat. Prod., 1989, 52, 774.

9 A. Gupta and S. R. Chaphalkar, J. HerbMed Pharmacol., 2016, 5, 120.

10 N. Askarian-Dehkordi, H. Shirzad, L. Salimzadeh, B. Zamanzad, R. Meshkate and M. Kiyani, J. HerbMed Pharmacol., 2014, 3, 125.

11 N. Desideri, S. Olivieri, M. L. Stein, R. Sgro, N. Orsi and C. Conti, Antiviral Chem. Chemother., 1997, 8, 545.

12 K. K. Purushothaman, K. Kalyani, K. Subramaniam and S. P. Shanmughanathan, Indian J. Chem., Sect. B: Org. Chem. Incl. Med. Chem., 1982, 21, 383.

13 D. D. McPherson, G. A. Cordell, D. D. Soejarto, J. M. Pezzuto and H. H. S. Fong, Phytochemistry, 1983, 22, 2835.

14 K. V. N. S. Srinivas, Y. K. Rao, I. Mahender and B. Das, Phytochemistry, 2003, 63, 789.

15 P. Zhao, Y. Iwamoto, I. Kouno, Y. Egami and H. Yamamoto, Phytochemistry, 2004, 65, 2455.

16 S. K. Roy, U. C. Agrahari, R. Gautam, A. Srivastava and S. M. Jachak, Nat. Prod. Res., 2012, 26, 690.

17 A. Foroumadi, A. Samzadeh-Kermani, S. Emami, G. Dehghan, M. Sorkhi, F. Arabsorkhi, M. R. Heidari, M. Abdollahi and A. Shafiee, Bioorg. Med. Chem. Lett., 2007, 17, 6764 .

18 (a) K. Eskandari and M. Rafieian-Kopaei, Chem. Heterocycl. Compd., 2016, 52, 158; (b) C. Li and F. Zhang, Tetrahedron Lett., 2017, 58, 1572; (c) H. Nagarajaiah, A. Mukhopadhyay and J. Narasimha-Moorthy, Tetrahedron Lett., 2016, 57, 5135.

19 S. Malhotra, V. K. Sharma and V. S. Parmar, J. Chem. Res., 1988, 179.

20 L. Farkas, A. Gottsegen and M. Nogradi, Tetrahedron, 1970, 26, 2787.

21 F. A. Davis and B. C. Chen, J. Org. Chem., 1993, 58, 1751. 
22 A. C. Jain and P. Paliwal, Indian J. Chem., Sect. B: Org. Chem. Incl. Med. Chem., 1989, 28, 416.

23 (a) K. Eskandari, B. Karami, M. Farahi and V. Mouzari, Tetrahedron Lett., 2016, 57, 487; (b) Y. Pourshojaei, A. Gouranourimi, S. Hekmat, A. Asadipour, S. RahmaniNezhad, A. Moradi, H. Nadri, F. Homayouni-Moghadam, S. Emami and A. Foroumadi, Eur. J. Med. Chem., 2015, 97, 181; (c) S. Rahmani-Nezhad, L. Khosravani, M. Saeedi, K. Divsalar, L. Firoozpour, Y. Pourshojaei, Y. Sarrafi, H. Nadri, A. Moradi, M. Mahdavi, A. Shafiee and A. Foroumadi, Synth. Commun., 2015, 45, 751; (d) S. Khodabakhshi, B. Karami, K. Eskandari and M. Farahi, Tetrahedron Lett., 2014, 55, 3753; (e) L. Poorali, B. Karami, K. Eskandari and M. Azizi, J. Chem. Sci., 2013, 125, 591.

24 (a) K. Eskandari, B. Karami and S. Khodabakhshi, J. Chem. Res., 2014, 38, 600; (b) K. Eskandari, B. Karami, S. Khodabakhshi and M. Farahi, Lett. Org. Chem., 2015, 12, 38; (c) B. Karami, R. Ferdosian and K. Eskandari, J. Chem. Res., 2014, 38, 41.

25 (a) K. Eskandari and B. Karami, Monatsh. Chem., 2016, 147, 2119; (b) B. Karami, K. Eskandari, Z. Zare and S. Gholipour, Chem. Heterocycl. Compd., 2014, 49, 1715; (c) K. Eskandari and B. Karami, Comb. Chem. High Throughput Screening, 2016, 19, 728.

26 (a) F. Mehrabi, Y. Pourshojaei, A. Moradi, M. Sharifzadeh, L. Khosravani, R. Sabourian, S. Rahmani-Nezhad, M. Mohammadi-Khanaposhtani, M. Mahdavi, A. Asadipour, H. R. Rahimi, S. Moghimi and A. Foroumadi, Future Med.
Chem., 2017, 9, 659; (b) S. Rahmani-Nezhad, M. Safavi, M. Pordeli, S. Kabudanian, L. Khosravani, Y. Pourshojaei, M. Mahdavi, S. Emami, A. Foroumadi and A. Shafiee, Eur. J. Med. Chem., 2014, 86, 562; (c) N. Azizi, F. Aryanasab and M. R. Saidi, Tetrahedron Lett., 2009, 50, 32; (d) M. R. Saidi, Y. Pourshojaei and F. Aryanasab, Synth. Commun., 2009, 39, 1109.

27 T. A. Nakibl, V. Bezjakl, M. J. Meeganz and R. Chandyl, Eur. J. Med. Chem., 1990, 25, 455.

28 N. Desideri, A. Bolasco, R. Fioravanti, L. P. Monaco, F. Orallo, M. Yanez, F. Ortuso and S. Alcaro, J. Med. Chem., 2011, 54, 2155.

29 N. Desideri, S. Olivieri, M. L. Stein, R. Sgro, N. Orsin and C. Conri, Antiviral Chem. Chemother., 1997, 8, 545.

30 S. W. Yee, L. Jarno, M. S. Goma, C. Elford, L. L. Ooi, M. P. Coogan, R. Mcclelland, R. I. Nicholson, B. A. J. Evans, A. Brancale and C. Simons, J. Med. Chem., 2005, 48, 7123.

31 X. Zong, J. Cai, J. Chen, C. Sun, L. Lib and M. Ji, RSC Adv., 2015, 5, 24814.

32 V. Siddaiah, M. Maheswara, C. V. Rao, S. Venkateswarlub and G. V. Subbaraju, Bioorg. Med. Chem. Lett., 2007, 17, 1288.

33 Y. Sun, J. Chen, X. Chen, L. Huang and X. Li, Bioorg. Med. Chem., 2013, 21, 7406.

34 Y. Jacquot, C. Byrne, A. Xicluna and G. Leclercq, Med. Chem. Res., 2013, 22, 681.

35 K. Koch and M. S. Biggers, J. Org. Chem., 1994, 59, 1216. 